\title{
COMPLETE CONTINUITY PROPERTIES OF BANACH SPACES ASSOCIATED WITH SUBSETS OF A DISCRETE ABELIAN GROUP
}

\author{
MANGATIANA A. ROBDERA \\ Eastern Mediterranean University, Gazimaguza, Via Mersin 10, Turkey \\ e-mail: robdera@yahoo.com \\ and PAULETTE SAAB \\ Department of Mathematics, University of Missouri, Columbia, MO 65211, U.S.A. \\ e-mail:paula@math.missouri.edu
}

(Received 20 July, 1999)

\begin{abstract}
We introduce and study the type I-, II-, and III- $\Lambda$-complete continuity property of Banach spaces, where $\Lambda$ is a subset of the dual group of a compact metrizable abelian group $G$.
\end{abstract}

2000 Mathematics Subject Classification. Primary 46E40, 46G10; Secondary 46B22.

1. Preliminaries. Throughout this paper $G$ will denote a compact metrizable abelian group. We denote by $\mathcal{B}(G)$ the $\sigma$-field of the Borel subsets of $G$, and by $\lambda$ a normalized Haar measure on $G$. The dual group of $G$ will be denoted by $\widehat{G}$.

If $X$ is a complex Banach space, then $B(X)$ will stand for the unit ball of the Banach space $X$, and $L^{1}(G, X)$ (resp. $L^{\infty}(G, X)$ ) denotes the Banach space of (all classes of) $\lambda$-Bochner integrable functions (resp. (all classes of) $X$-valued $\lambda$-measurable functions that are essentially bounded) on $G$ with values in $X$. The space of all continuous $X$-valued functions on $G$ will be denoted by $C(G, X)$. If $X=\mathbb{C}$, then $L^{1}(G, X), L^{\infty}(G, X)$ and $C(G, X)$ will be denoted by $L^{1}(G), L^{\infty}(G)$ and $C(G)$ respectively.

The symbol $\mathcal{M}^{1}(G, X)$ will be used to denote the space of countably additive $X$-valued measures that are of bounded variation, so $\mu \in \mathcal{M}^{1}(G, X)$ if the quantity

$$
\|\mu\|_{1}=\sup \left\|\sum_{A \in \pi} \frac{\mu(A)}{\lambda(A)} \chi_{A}\right\|_{1}
$$

is finite, where the supremum is taken over all finite partitions $\pi$ consisting of Borel subsets of $G$. Here for each Borel subset $A$ of $G, \chi_{A}$ denotes the characteristic function of $A$. An $X$-valued measure $\mu$ on $G$ such that for every Borel subset $A$ of $G$, $\|\mu(A)\|_{X} \leq c \lambda(A)$, for some positive contant $c$, is said to be of bounded average range. The infinimum of such constant $c$ defines a norm on the space of vector measures and is denoted by $\|\mu\|_{\infty}$. The Banach space of all $X$-valued countably additive measures on $G$ with $\|\mu\|_{\infty}<\infty$ is denoted by $\mathcal{M}^{\infty}(G, X)$.

If $X$ and $Y$ are Banach spaces, then $\mathcal{L}(X, Y)$ will denote the Banach space of all bounded linear operators from $X$ to $Y$.

A bounded linear operator $T: X \longrightarrow Y$ is said to be completely continuous (also called Dunford-Pettis) if it maps weakly convergent sequences in the Banach space $X$ into norm convergent sequences in the Banach space $Y$. Recall that a Banach 
space $X$ has the complete continuity property $(\mathrm{CCP})$ if every bounded linear operator $T: L^{1}(G) \rightarrow X$ is completely continuous.

2. The $\Lambda$-complete continuity property types. Let $\Lambda$ be a subset of the dual group of $G$, and $\Lambda^{\prime}=\{\gamma \in \widehat{G}, \bar{\gamma} \notin \Lambda\}$, where $\bar{\gamma}$ is the conjugate character of $\gamma$. For $\gamma \in \widehat{G}$, $f \in L^{1}(G, X)$, the Fourier coefficient of $f$ at $\gamma$ is defined by

$$
\hat{f}(\gamma)=\int_{G} f(t) \bar{\gamma}(t) d \lambda(t) .
$$

More generally, if $\mu \in \mathcal{M}^{1}(G, X)$, the Fourier coefficient of $\mu$ at $\gamma$ is defined by

$$
\widehat{\mu}(\gamma)=\int_{G} \bar{\gamma}(t) d \mu(t) .
$$

In what follows we shall use the following:

$$
\begin{array}{ll}
L_{\Lambda}^{1}(G, X) & =\left\{f \in L^{1}(G, X): \widehat{f}(\gamma)=0 \text { for } \gamma \notin \Lambda\right\} \\
\mathcal{M}_{\Lambda}^{1}(G, X) & =\left\{\mu \in \mathcal{M}^{1}(G, X): \widehat{\mu}(\gamma)=0 \text { for } \gamma \notin \Lambda\right\} \\
\mathcal{M}_{\Lambda a c}^{1}(G, X) & =\left\{\mu \in \mathcal{M}^{1}(G, X): \mu \text { is } \lambda \text {-continuous and } \widehat{\mu}(\gamma)=0 \text { for } \gamma \notin \Lambda\right\} \\
\mathcal{C}_{\Lambda}(G, X) & =\{f \in C(G, X): \widehat{f}(\gamma)=0 \text { for } \gamma \notin \Lambda\} .
\end{array}
$$

Each element of $L_{\Lambda}^{1}(G, X)$ (resp. $\left.\mathcal{M}_{\Lambda}^{1}(G, X)\right)$ will be termed as $\Lambda$-function (resp. $\Lambda$ measure). For the particular case where the Banach space $X=\mathbb{C}, L_{\Lambda}^{1}(G, \mathbb{C})$, $\mathcal{M}_{\Lambda}^{1}(G, \mathbb{C})$, and $\mathcal{C}_{\Lambda}(G, \mathbb{C})$ will be simply denoted by $L_{\Lambda}^{1}(G), \mathcal{M}_{\Lambda}^{1}(G)$, and $\mathcal{C}_{\Lambda}(G)$ respectively.

In what follows we shall introduce types of complete continuity property associated to a subset $\Lambda$ of the dual group $\widehat{G}$. These properties can be seen as the complete continuity counterpart of the types of Radon-Nikodým properties introduced by G. A. Edgar in [6], and P. Dowling in [4]. We recall that a Banach space $X$ is said to have type $I$ - $\Lambda$-Radon-Nikodým property (I- $\Lambda$-RNP), (resp. II- $\Lambda$-Radon-Nikodým property (II- $\Lambda$-RNP)) if every $X$-valued $\Lambda$-measure of bounded average range (resp.; of bounded variation) is differentiable (i.e. $\mathcal{M}_{\Lambda}^{\infty}(G, X)=L_{\Lambda}^{\infty}(G, X)$ (resp.; $\left.\left.\mathcal{M}_{\text {Aac }}^{1}(G, X)=L_{\Lambda}^{1}(G, X)\right)\right)$ [4]. An element $\mu$ of $\mathcal{M}^{1}(G, X)$ is said to have a relatively compact range if the set $\{\mu(A): A \in \mathcal{B}(G)\}$ is relatively compact in $X$.

Definition 1. Let $\Lambda$ be a subset of the dual group of a compact metrizable abelian group $G$. A Banach space $X$ is said to have type I- $\Lambda$-complete continuity property $(\mathrm{I}-\Lambda$-CCP) if every $X$-valued $\Lambda$-measure of bounded average range has a relatively compact range.

Definition 2. A Banach space is said to have type II- $\Lambda$-complete continuity property (II- $\Lambda$-CCP) if every $X$-valued $\lambda$-continuous $\Lambda$-measure of bounded variation has relatively compact range.

It is immediate that the type I- $\Lambda$-RNP (resp; II- $\Lambda$-RNP) implies the type I- $\Lambda$-CCP (resp; II- $\Lambda$-CCP). Moreover, since every element of $\mathcal{M}_{\Lambda}^{\infty}(G, X)$ is in particular an element of $\mathcal{M}_{\Lambda a c}^{1}(G, X)$, one easily notices that type II- $\Lambda$-CCP implies type I- $\Lambda$-CCP. 
Every member $\mu \in \mathcal{M}_{A}^{\infty}(G, X)$ naturally defines a bounded linear operator $T: L^{1}(G) \longrightarrow X$ by $T(f)=\int_{G} f d \mu$, for all $f \in L^{1}(G)$. A simple computation shows that $T(\bar{\gamma})=\widehat{\mu}(\gamma)=0$ for all $\gamma \notin \Lambda$. Bounded linear operators from $L^{1}(G)$ into a Banach space $X$ with the property $T(\bar{\gamma})=0$ for $\gamma \notin \Lambda$ will be called $\Lambda$-operators. Conversely, to a $\Lambda$-operator $T$ from $L^{1}(G)$ into a Banach space $X$ one can associate an element $\mu$ of $M_{\Lambda}^{\infty}(G, X)$ by $\mu(A)=T\left(\chi_{A}\right)$ for every $A \in \mathcal{B}(G)$. This leads us to the following:

THEOREM 2.1. Let $\Lambda$ be a subset of the dual group of a compact metrizable abelian group $G$. A Banach space $X$ has type I- $\Lambda$-CCP if and only if every 1 -operator $T: L^{1}(G) \longrightarrow X$ is a completely continuous operator.

One notices that for $\Lambda=\widehat{G}$, the I- $\Lambda$-CCP type and the II- $\Lambda$-CCP coincide with the complete continuity property. Also if $\Lambda_{1} \subset \Lambda_{2}$ then type I- $\Lambda_{2}-\mathrm{CCP}$ (resp; II- $\Lambda_{2}$ $\mathrm{CCP}$ ) implies type I- $\Lambda_{1}-\mathrm{CCP}$ (resp; II- $\left.\Lambda_{2}-\mathrm{CCP}\right)$. In particular:

REMARK 2.2. If a Banach space $X$ has the complete continuity property then it has the type I- $\Lambda$-CCP and II- $\Lambda$-CCP for any $\Lambda \subset \widehat{G}$.

It is known that the Banach space $L^{1}(G)$ fails the complete continuity property; however we will see that $L^{1}(G)$ has I- $\Lambda$-CCP for some $\Lambda \subset \widehat{G}$. The first example of a Banach space failing the I- $\Lambda$-CCP is provided by:

Proposition 2.3. Let $\Lambda$ be an infinite subset of the dual group of a compact metrizable abelian group $G$. The sequence space $c_{0}$ fails $\mathrm{I}-\Lambda$-CCP.

Proof. To see this, let $\left(\gamma_{n}\right)_{n \in \mathbb{N}}$ be an enumeration of the elements of $\Lambda$. Define an operator $T: L^{1}(G) \longrightarrow c_{0}$ by

$$
T f=\left(\int_{G} f(t) \gamma_{n}(t) d \lambda(t)\right)_{n \in \mathbb{N}}
$$

for all $f \in L^{1}(G)$. Then $T$ is a bounded linear operator with $T(\bar{\gamma})=0$ for $\gamma \notin\left(\gamma_{n}\right)_{n \in \mathbb{N}}$. Since for every function $f \in L^{1}(G), \quad\left(\widehat{f}(\gamma)=\int_{G} f(t) \bar{\gamma}(t) d \lambda(t)\right)_{\gamma \in \widehat{G}} \in c_{0}(\widehat{G})$ (see for example [13]), it is clear that the sequence $\left(\bar{\gamma}_{n}\right)_{n \in \mathbb{N}}$ is weakly null; however $\left\|T\left(\bar{\gamma}_{n}\right)\right\|_{c_{0}}=1$ for $n=1,2, \ldots$. Thus the operator $T$ is a $\Lambda$-operator which is not completely continuous.

It is apparent that if a Banach space $X$ has I- $\Lambda$-CCP (resp. II- $\Lambda$-CCP) type then so does each one of its subspaces. On the other hand, since the group $G$ is compact metrizable, $\mathcal{B}(G)$ is countably generated, one sees that the I- $\Lambda$-CCP (resp. II- $\Lambda$-CCP) type is separably determined, i.e.:

THEOREM 2.4. Let $\Lambda$ be a subset of the dual group of a compact metrizable abelian group $G$. A Banach space $X$ has type I- $\Lambda$-CCP (resp. II- $\Lambda$-CCP) if and only if so has each one of its separable subspaces.

Also recall that a subset $\Lambda$ of $\widehat{G}$ is said to be a Riesz set if $\mathcal{M}_{\Lambda}^{1}(G)=L_{\Lambda}^{1}(G)(\mathrm{cf}$. [9]), and $\Lambda$ is a Sidon set if $C_{\Lambda}(G)=\ell^{1}(\Lambda)$. It can be deduced from [4] and [11] that 
types I- $\Lambda$-RNP and II- $\Lambda$-RNP are the same for Banach lattices provided $\Lambda$ is Riesz, and they are equivalent to the non containment of isomorphic copies of $c_{0}$. In view of Proposition 2.3, we also have the following results.

TheOREM 2.5. Let $\Lambda$ be a Riesz subset of $\widehat{G}$. Then the following properties are equivalent for a Banach lattice $X$ :
(a) $X$ has type II- $\Lambda$-RNP;
(b) $X$ has type I- $\Lambda$-RNP;
(c) $X$ has type II- $\Lambda$-CCP;
(d) X has type I- $\Lambda$-CCP;
(e) $X$ contains no subspaces isomorphic to $c_{0}$.

We also have the following result which it can be deduced from a result of [5].

Theorem 2.6. Let $\Lambda$ be a Sidon set of $\hat{G}$. The following properties of an arbitrarily Banach space $X$ are equivalent:
(a) $X$ has type II- $\Lambda$-RNP;
(b) X has type I- $\Lambda$-RNP;
(c) $X$ has type II- $\Lambda$-CCP;
(d) $X$ has type I- $\Lambda$-CCP;
(e) $X$ contains no subspace isomorphic to $c_{0}$.

3. Characterizations of the $\Lambda$-CCP types. For a compact metrizable abelian group $G$, a sequence $\left(i_{n}\right)_{n \in \mathbb{N}}$ of measurable functions $i_{n}: G \longrightarrow \mathbb{R}$ is called a good approximate identity on $G$ if

(1) $i_{n} \geq 0$ for all $n \in \mathbb{N}$,

(2) $\int_{G} i_{n}(t) d \lambda(t)=1$ for all $n \in \mathbb{N}$,

(3) $\sum_{\gamma \in \widehat{G}} \widehat{i_{n}}(\gamma)<\infty$ for all $n \in \mathbb{N}$, and

(4) $\lim _{n \rightarrow \infty} \int_{U} i_{n}(t) d \lambda(t)=1$ for every neighbourhood $U$ of the identity element of $G$.

We recall that for any compact metrizable abelian group $G$, a good approximate identity always exists on $G$ (see for example [6], [8] or [13]).

For a Banach space $X$, and for an element $f$ of $L^{1}(G, X)$ the Pettis-norm of $f$ is given by

$$
\|f\| \mid=\sup \left\{\int_{G}\left|x^{*} f\right| d \lambda: x^{*} \in X^{*},\left\|x^{*}\right\| \leq 1\right\} .
$$

We say that a sequence $\left(f_{n}\right)$ of elements of $L^{1}(G, X)$ is Pettis-Cauchy if it is a Cauchy sequence for the Pettis-norm.

In what follows we shall give characterizations of the I- $\Lambda$-CCP and II- $\Lambda$-CCP properties. Our results should be compared to the following theorems of [4] and [6] which characterize the different types of $\Lambda$-RNP spaces:

THEOREM 3.1. (Edgar). Let $G$ be a compact metrizable abelian group, let $\Lambda \subset \widehat{G}$ and let $\left(i_{n}\right)_{n \in \mathbb{N}}$ be a good approximate identity on $G$. Then the following properties are equivalent for a Banach space $X$ : 
(a) $X$ has I- $\Lambda$-RNP;

(b) if $\left(a_{\gamma}\right)_{\gamma \in \Lambda} \subset X$ and $\left(f_{n}=\sum_{\gamma \in \Lambda} \widehat{i_{n}}(\gamma) a_{\gamma} \gamma\right)_{n \in \mathbb{N}}$ is bounded in $L_{\Lambda}^{\infty}(G, X)$, then the sequence $\left(f_{n}\right)_{n \in \mathbb{N}}$ converges in $L^{1}(G, X)$-norm.

THeOREM 3.2. (Dowling). Let $G$ be a compact metrizable abelian group, let $\Lambda$ be a Riesz subset of $\widehat{G}$ and let $\left(i_{n}\right)_{n \in \mathbb{N}}$ be a good approximate identity on $G$. Then the following are equivalent for a Banach space $X$ :

(a) $X$ has II- $\Lambda$-RNP;

(b) if $\left(a_{\gamma}\right)_{\gamma \in \Lambda} \subset X$ and $\left(f_{n}=\sum_{\gamma \in \Lambda} \widehat{i_{n}}(\gamma) a_{\gamma} \gamma\right)_{n \in \mathbb{N}}$ is bounded in $L_{\Lambda}^{1}(G, X)$, then the sequence $\left(f_{n}\right)$ converges in $L^{1}(G, X)$-norm.

THEOREM 3.3. Let $G$ be a compact metrizable abelian group, let $\Lambda \subset \widehat{G}$ and let $\left(i_{n}\right)_{n \in \mathbb{N}}$ be a good approximate identity on $G$. Then the following properties are equivalent for a Banach space $X$ :

(a) $X$ has I- $\Lambda$-CCP;

(b) if $\left(a_{\gamma}\right)_{\gamma \in \Lambda} \subset X$ and $\left(f_{n}=\sum_{\gamma \in \Lambda} \widehat{i_{n}}(\gamma) a_{\gamma} \gamma\right)_{n \in \mathbb{N}}$ is bounded in $L^{\infty}(G, X)$, then the sequence $\left(f_{n}\right)_{n \in \mathbb{N}}$ is Pettis-Cauchy.

Proof. (a) $\Rightarrow$ (b). Let $\left(a_{\gamma}\right)_{\gamma \in \Lambda} \subset X$ and suppose the sequence $\left(f_{n}=\right.$ $\left.\sum_{\gamma \in \Lambda} \widehat{i_{n}}(\gamma) a_{\gamma} \gamma\right)_{n \in \mathbb{N}}$ is bounded in $L^{\infty}(G, X)$. We want to show that

$$
\lim _{n, m}\left\|f_{n}-f_{m}\right\|=\lim _{n, m} \sup \left\{\int_{G}\left|x^{*} f_{n}-x^{*} f_{m}\right| d \lambda, x^{*} \in X^{*},\left\|x^{*}\right\| \leq 1\right\}=0 .
$$

To this end, we define, for each $n \in \mathbb{N}$, the operator $T_{n}: L^{1}(G) \longrightarrow X$ by $T_{n}(f)=$ $\int_{G} f f_{n} d \lambda$, for all $f \in L^{1}(G)$. Then $\left\|T_{n}\right\|=\left\|f_{n}\right\|_{L^{\infty}(G, X)}$, for all $n \in \mathbb{N}$. Thus $\sup _{n}\left\|T_{n}\right\|<\infty$. Let $\left(T_{n_{\alpha}}\right)$ be a subnet of $\left(T_{n}\right)$ that converges to an operator $T: L^{1}(G) \longrightarrow X^{* *}$ in the weak* operator topology. In particular, for each $\gamma \in \widehat{G}$ and each $x^{*} \in B\left(X^{*}\right)$,

$$
<T(\bar{\gamma}), x^{*}>=\lim _{n_{\alpha}} \int_{G} \bar{\gamma}(s) x^{*} f_{n_{\alpha}}(s) d \lambda(s)=\lim _{n_{\alpha}} x^{*} \widehat{f}_{n_{\alpha}}(\gamma) .
$$

Thus $T(\bar{\gamma})=a_{\gamma}$ if $\gamma \in \Lambda$ and $T(\bar{\gamma})=0$ if $\gamma \notin \Lambda$. Since the characters form a total subset of $L^{1}(G)$, it follows that $T$ is a bounded linear $\Lambda$-operator from $L^{1}(G)$ into $X$. Hence by our assumption, it is a completely continuous operator. Since the unit ball of $L^{\infty}(G)$ is relatively weakly compact in $L^{1}(G)$, the operator $S=\left.T\right|_{L^{\infty}(G)}$ is compact.

For every function $g \in L^{\infty}(G)$, and for each $x^{*} \in X^{*}$, it is clear that

$$
\begin{aligned}
<S^{*} x^{*}, g> & =<x^{*}, T g> \\
& =\lim _{n_{\alpha}} x^{*} \int_{G} f_{n_{\alpha}} g d \lambda \\
& =\lim _{n_{\alpha}} \int_{G} x^{*} f_{n_{\alpha}} g d \lambda .
\end{aligned}
$$

Equations 3.1 show that $S^{*} x^{*}=$ weak- $\lim x^{*} f_{n_{\alpha}}$, and hence it shows that $S^{*}$ takes its values in $L^{1}(G)$. 
Now let $R_{n}: L^{1}(G) \longrightarrow L^{1}(G)$ denote the convolution operator defined by $R_{n} f=i_{n} * f$ for all $f \in L^{1}(G)$, for each $n \in \mathbb{N}$. Since for each $f \in L^{1}(G)$, the sequence $\left(R_{n}(f)\right)$ converges to $f \in L^{1}(G)$ (see for example [13]), the sequence of operators $\left(R_{n}\right)$ converges uniformly on compact subsets of $L^{1}(G)$. For $x^{*} \in X^{*},\left\|x^{*}\right\| \leq 1$, one has

$$
\begin{aligned}
R_{n} S^{*} x^{*} & =\sum_{\gamma \in \widehat{G}} \widehat{i_{n}}(\gamma) \widehat{S^{*} x^{*}}(\gamma) \gamma \\
& =\sum_{\gamma \in \widehat{G}} \widehat{i_{n}}(\gamma) x^{*} S(\bar{\gamma}) \gamma \\
& =\sum_{\gamma \in \Lambda} \widehat{i_{n}}(\gamma) x^{*} a_{\gamma} \gamma=x^{*} f_{n}
\end{aligned}
$$

Therefore,

$$
\lim _{n, m}\left\|f_{n}-f_{m}\right\|=\lim _{n, m} \sup \left\{\left\|\left(R_{n}-R_{m}\right) S^{*} x^{*}\right\|: x^{*} \in X^{*},\left\|x^{*}\right\| \leq 1\right\} .
$$

The compactness of $S$ now implies that this limit is 0 as desired.

(b) $\Rightarrow$ (a) Let $T: L^{1}(G) \longrightarrow X$ be a $\Lambda$-operator. Consider the sequence of functions $\left(f_{n}=\sum_{\gamma \in \widehat{G}} \widehat{i_{n}}(\gamma) T(\bar{\gamma}) \gamma\right)_{n \geq 1}$. One has, for each $t \in G$, and for $n \in \mathbb{N}$

$$
f_{n}(t)=\sum_{\gamma \in \widehat{G}} \widehat{i_{n}}(\gamma) T(\bar{\gamma}) \gamma(t)=T\left(\sum_{\gamma \in \widehat{G}} \widehat{i_{n}}(\gamma) \gamma(t-.)\right)=T\left(i_{n}(t-.)\right) .
$$

Then $\left\|f_{n}\right\|_{L^{\infty}(G, X)} \leq\|T\|$, for all $n \in \mathbb{N}$. Hence $\left(f_{n}\right)$ is Pettis-Cauchy by our assumption.

Conversely, let $T_{n} \in \mathfrak{Q}\left(L^{1}(G), X\right)$ be the bounded linear operator defined by $T_{n} f=\int_{G} f f_{n} d \lambda$, for every $f \in L^{1}(G)$, and denote by $j_{\infty}$ the natural injection of $L^{\infty}(G)$ into $L^{1}(G)$. Consider the composition operator $S_{n}=T_{n} j_{\infty}$, for each $n \in \mathbb{N}$. Since $T_{n}$ is completely continuous and the unit ball of $L^{\infty}(G)$ is relatively weakly compact in $L^{1}(G)$, the operator $S_{n}$ is compact. For $x^{*} \in X^{*}$, and for every $f \in L^{\infty}(G)$

$$
S_{n}^{*} x^{*}(f)=x^{*} S_{n}(f)=x^{*} T_{n} j_{\infty}(f)=x^{*} \int_{G} f f_{n} d \lambda=\int_{G} f x^{*} f_{n} d \lambda .
$$

Thus $S_{n}^{*} x^{*}=x^{*} f_{n}$, for each $n \in \mathbb{N}$. Hence, for $n, m \in \mathbb{N}$,

$$
\begin{aligned}
\left\|S_{n}-S_{m}\right\| & =\left\|S_{n}^{*}-S_{m}^{*}\right\| \\
& =\sup \left\{\left\|\left(S_{n}^{*}-S_{m}^{*}\right)\left(x^{*}\right)\right\|_{1} ; x^{*} \in X^{*},\left\|x^{*}\right\| \leq 1\right\} \\
& =\sup \left\{\left\|x^{*} f_{n}-x^{*} f_{m}\right\|_{1} ; x^{*} \in X^{*},\left\|x^{*}\right\| \leq 1\right\} \\
& =\left\|f_{n}-f_{m}\right\| .
\end{aligned}
$$

Thus the sequence $\left(S_{n}\right)_{n \geq 1}$ is Cauchy in $\mathfrak{2}\left(L^{\infty}(G), X\right)$, and hence it converges to an operator $S: L^{\infty}(G) \longrightarrow X$. Since each operator $S_{n}$ is compact for each $n=1,2, \ldots$, so is the operator $S$. 
On the other hand, for $f \in L^{\infty}(G)$, one has

$$
\begin{aligned}
S_{n} f=T_{n} j_{\infty} f & =\int_{G} f \sum_{\gamma \in \widehat{G}} \widehat{i_{n}}(\gamma) T(\bar{\gamma}) \gamma d \lambda \\
& =\sum_{\gamma \in \widehat{G}} \widehat{i_{n}}(\gamma) \widehat{f}(\bar{\gamma}) T \bar{\gamma} \\
& =T\left(\sum_{\gamma \in \widehat{G}} \widehat{i_{n}}(\gamma) \widehat{f}(\bar{\gamma}) \bar{\gamma}\right) \\
& =T\left(i_{n}^{\prime} * f\right)
\end{aligned}
$$

where $i_{n}^{\prime}(t)=i_{n}(-t)$, for $t \in G$ and for all $n \in \mathbb{N}$. Thus

$$
\left\|\left(T-T_{n}\right)(f)\right\|=\left\|T\left(f-i_{n}^{\prime} * f\right)\right\| \leq\|T\|\left\|f-i_{n}^{\prime} * f\right\|_{L^{1}(G)},
$$

for any positive integer $n$. It follows that the sequence of operators $\left(T_{n}\right)_{n>1}$ converges to $T$ on $L^{\infty}(G)$, in the strong operator topology. Consequently, we have $T \equiv S$ on $L^{\infty}(G)$. Therefore, we can conclude that the restriction of the operator $T$ on $L^{\infty}(G)$ is compact. This shows that the operator $T$ is indeed completely continuous.

The next theorem gives a characterization of the type II- $\Lambda$-CCP. This result can naturally be compared to the characterization theorem of the type II- $\Lambda$-RNP as given in [4] (see Theorem 3.2 above).

THEOREM 3.4. Let $G$ be a compact metrizable abelian group, let $\Lambda$ be a Riesz subset of $\widehat{G}$ and let $\left(i_{n}\right)_{n \in \mathbb{N}}$ be a good approximate identity on $G$. Then the following are equivalent for a Banach space $X$ :

(a) $X$ has II- $\Lambda$-CCP;

(b) if $\left(a_{\gamma}\right)_{\gamma \in \Lambda} \subset X$ and $\left(f_{n}=\sum_{\gamma \in \Lambda} \widehat{i_{n}}(\gamma) a_{\gamma} \gamma\right)_{n \in \mathbb{N}}$ is bounded in $L^{1}(G, X)$, then the sequence $\left(f_{n}\right)_{n \in \mathbb{N}}$ is Pettis-Cauchy.

Proof. (a) $\Rightarrow$ (b) Let $\left(a_{\gamma}\right)_{\gamma \in \Lambda} \subset X$ and assume that $\left(f_{n}=\sum_{\gamma \in \Lambda} \widehat{i_{n}}(\gamma) a_{\gamma} \gamma\right)_{n \in \mathbb{N}}$ is bounded in $L^{1}(G, X)$. For each $n \geq 1$, let $\mu_{n} \in \mathcal{M}^{1}(G, X)$ be defined by

$$
\mu_{n}(A)=\int_{G} \chi_{A}(t) f_{n}(t) d \lambda(t),
$$

for each $A \in \mathcal{B}(A)$. Then $\left\|\mu_{n}\right\|_{1}=\left\|f_{n}\right\|_{1}$, for each $n \geq 1$.

Consider the space $\mathcal{M}^{1}\left(G, X^{* *}\right)$. It is well known [2], that $\mathcal{M}^{1}\left(G, X^{* *}\right)$ is isometrically isomorphic to the dual space $\mathcal{C}\left(G, X^{*}\right)^{*}$. Since by our assumption the sequence $\left(\mu_{n}\right)$ is bounded in $\mathcal{M}^{1}(G, X)$, it is also bounded in $\mathcal{M}^{1}\left(G, X^{* *}\right)$. Let $\left(\mu_{n_{\alpha}}\right)$ be a subnet of $\left(\mu_{n}\right)$ that converges to an element $v$ in $\mathcal{M}^{1}\left(G, X^{* *}\right)$ in the weak* topology. Then in particular for each character $\gamma \in \widehat{G}$, and for each element $x^{*} \in X^{*}$, we have

$$
\widehat{v}(\gamma) x^{*}=\lim _{n_{\alpha}} \int_{G} \bar{\gamma} x^{*} f_{n_{\alpha}} d \lambda=x^{*}\left(\lim _{n_{\alpha}} \widehat{f}_{n_{\alpha}}(\gamma)\right) .
$$


Thus

$$
\widehat{v}(\gamma)=\left\{\begin{array}{cl}
a_{\gamma}, & \text { if } \quad \gamma \in \Lambda, \\
0, & \text { if } \quad \gamma \notin \Lambda .
\end{array}\right. \text { and }
$$

Since the characters form a total subset of $\mathcal{C}(G)$, it follows that the mapping $x^{*} \longrightarrow v(\cdot) x^{*}$ of $X^{*}$ into $\mathcal{C}(G)^{*}$ is weak ${ }^{*}$ to weak ${ }^{*}$ continuous. Therefore, we can define a bounded linear operator $T: \mathcal{C}(G) \longrightarrow X$ by $x^{*} T(f)=\int_{G} f d\left(x^{*} v\right)$, for each $f \in \mathcal{C}(G)$ and for each $x^{*} \in X^{*}$ [2, Theorem 1]. Since by our assumption $X$ has II- $\Lambda$-CCP, $X$ contains no isomorphic copy of $c_{0}$. Thus the operator $T$ is weakly compact and consequently the measure $v$ takes its values in $X[2$, p. 238]. Since $\hat{v}(\gamma)=0$ if $\gamma \notin \Lambda$, and $\Lambda$ is a Riesz set, then $v$ is absolutely continuous with respect to Haar measure on $G$. Thus, by our assumption, the measure $v$ has relatively compact range and hence the operator $T$ is compact.

On the other hand, it is easily seen that $\lim _{n} x^{*} f_{n}$ exists in $L^{1}(G)$ and that

$$
<\lim _{n} x^{*} f_{n}, f>=<x^{*}, T f>=<T^{*} x^{*}, f>,
$$

for each $x^{*} \in X^{*}$ and for each $f \in \mathcal{C}(G)$. That is, the adjoint operator of the operator $T$ is given by $T^{*} x^{*}=\lim _{n} x^{*} f_{n}$, for each $x^{*} \in X^{*}$, and thus $T^{*} x^{*} \in L^{1}(G)$. From here we just repeat the last part of the proof of the implication (a) $\Rightarrow(b)$ of the Theorem 3.3. This establishes (a) $\Rightarrow$ (b). (b) $\Rightarrow$ (a) Let $\mu \in \mathcal{M}_{\text {Aac }}^{1}(G, X)$. Set $\widehat{\mu}(\gamma)=a_{\gamma}, \gamma \in \widehat{G}$ and let $f_{n}=\sum_{\gamma \in \widehat{G}} \widehat{i_{n}}(\gamma) a_{\gamma} \gamma$.
Thus for $n \in \mathbb{N}$, and for $t \in G$,

$$
\begin{aligned}
i_{n} * \mu(t) & =\int_{G} i_{n}(t-s) d \mu(s) \\
& =\int_{G} \sum_{\gamma \in \widehat{G}} \widehat{i_{n}}(\gamma) \gamma(t) \bar{\gamma}(s) d \mu(s) \\
& =\sum_{\gamma \in \widehat{G}} \widehat{i_{n}}(\gamma) \widehat{\mu}(\gamma) \gamma(t) \\
& =\sum_{\gamma \in \widehat{G}} \widehat{i_{n}}(\gamma) a_{\gamma} \gamma(t)=f_{n}(t) .
\end{aligned}
$$

Therefore $\left\|f_{n}\right\|_{L^{1}(G, X)}=\left\|i_{n} * \mu\right\|_{L^{1}(G, X)} \leq\|\mu\|_{1}$, for all $n \in \mathbb{N}$. Thus the sequence $\left(f_{n}\right)$ is Pettis-Cauchy.

For each $n \in \mathbb{N}$, let $\mu_{n}=f_{n} \cdot \lambda$. For $n, m \in \mathbb{N}$, and $E \in \mathcal{B}(G)$,

$$
\left\|\mu_{n}(E)-\mu_{m}(E)\right\| \leq\left\|f_{n}-f_{m}\right\| .
$$

Thus there exists a set function $v: \mathcal{B}(G) \longrightarrow X$ such that $v(E)=\lim _{n} \mu_{n}(E)$ uniformly on $\mathcal{B}(G)$. An appeal to Vitali-Hahn-Saks' Theorem (cf. [2]), shows that $v$ is $\lambda$ continuous.

Now since by construction the $\mu_{n}$ have relatively compact ranges, we claim that $v$ also has the same property. Indeed, given $\epsilon>0$, there exists $n_{\epsilon} \in \mathbb{N}$ large enough such that 


$$
\left\|v(E)-\mu_{n_{\epsilon}}(E)\right\|<\epsilon / 3 \text {, for } E \in \mathcal{B}(G) .
$$

Thus it follows that

$$
\{v(E) ; E \in \mathcal{B}(G)\} \subset\left\{\mu_{n_{\epsilon}}(E) ; E \in \mathcal{B}(G)\right\}+\epsilon B(X),
$$

where $B(X)$ denotes the unit ball of $X$. As mentioned above, we have that the set $\left\{\mu_{n_{\epsilon}}(E) ; E \in \mathcal{B}(G)\right\}$ is relatively compact for each $\epsilon>0$, and so is $\{\nu(E)$; $E \in \mathcal{B}(G)\}$ by a standard argument. This proves our claim. Finally, for $\gamma \in \widehat{G}$, we have

$$
\widehat{v}(\gamma)=\lim _{n} \int_{G} \bar{\gamma} f_{n} d \lambda=\lim _{n} \widehat{f_{n}}(\gamma)=a_{\gamma}=\widehat{\mu}(\gamma) .
$$

We conclude that $\mu=v$ and thus $\mu$ has relatively compact range.

REMARK 3.5. The hypothesis that $\Lambda$ is a Riesz set was only needed in the implication $(\mathrm{a}) \Rightarrow(\mathrm{b})$.

Finally, let us introduce the following type of $\Lambda$-CCP which has very interesting properties as did its Radon-Nikodým counterpart [4].

Defintion 3. Let $\Lambda$ be a subset of the dual group of a compact metrizable abelian group $G$. A Banach space $X$ is said to have type III- $\Lambda$-complete continuity property (III- $\Lambda$-CCP), if every absolutely summing operator [3] $T: \mathcal{C}(G) \longrightarrow X$ with $T \equiv 0$ on $\mathcal{C}_{\Lambda^{\prime}}(G)$ is compact.

The following two interesting results were shown in [4].

Proposition 3.6 (Dowling). Let $\Lambda$ be a Riesz subset of the dual group of a compact metrizable abelian group $G$. Then a Banach space $X$ has type II- $\Lambda$-RNP if and only if it has III- $\Lambda$-RNP.

Proposition 3.7 (Dowling). Let $\Lambda$ be a non Riesz subset of the dual group $\widehat{G}$ of a compact metrizable abelian group $G$. Then a Banach space $X$ has type III- $\Lambda$-RNP if and only if it has the Radon-Nikodym property.

As it was shown in the above results, the next two propositions show that the type III- $\Lambda$-CCP is not an isolated property. It coincides with either of type II- $\Lambda$-CCP or CCP depending on whether or not $\Lambda$ is a Riesz set.

First, it is known and easy to see that if $\Lambda$ is Riesz then $\mathcal{M}_{\Lambda}^{1}(G, X)=$ $\mathcal{M}_{A a c}^{1}(G, X)$, for any Banach space $X$. Consequently, we obtain the following result.

Proposition 3.8. Let $\Lambda$ be a Riesz subset of the dual group of a compact metrizable abelian group $G$. Then a Banach space $X$ has type II- $\Lambda$-CCP if and only if it has III- $\Lambda$-CCP.

Proof. First note that type III- $\Lambda$-CCP implies type II- $\Lambda$-CCP for any subset $\Lambda \subset \hat{G}$. To see this, assume that the Banach space $X$ has type III- $\Lambda$-CCP and let $\mu$ be in $\mathcal{M}_{A a c}^{1}(G, X)$. A simple computation shows that the integration operator 
$T: \mathcal{C}(G) \longrightarrow X$ defined by $T(f)=\int_{G} f d \mu$, for all $f \in \mathcal{C}(G)$ is absolutely summing and $T(\gamma)=\int_{G} \gamma d \mu=\widehat{\mu}(\bar{\gamma})=0$ for every $\gamma \in \Lambda^{\prime}$. Therefore $T$ is compact. Since for each Borel subset $A$ of $G$

$$
\mu(A)=T^{* *}\left(\chi_{A}\right),
$$

where $\chi_{A}$ denotes the characteristic function of $A$. It follows that the measure $\mu$ has relatively compact range. Therefore $X$ has type II- $\Lambda$-CCP.

For the converse, suppose the Banach space $X$ has type II- $\Lambda$-CCP and let $T: \mathcal{C}(G) \longrightarrow X$ be an absolutely summing operator such that $T \equiv 0$ on $\mathcal{C}_{\Lambda^{\prime}}(G)$. Let $\mathfrak{\wp}: \mathcal{B}(G) \rightarrow X^{* *}$ be the vector measure representing the operator $T$, i.e. for each Borel subset $A$ of $G$,

$$
\mathfrak{\Im}(A)=T^{* *}\left(\chi_{A}\right) .
$$

Since $T$ is absolutely summing, it is in particular weakly compact and hence its representing measure $\mathfrak{\gamma}$ takes its values in $X$. On the other hand, $\mathfrak{\gamma}(\gamma)=T(\bar{\gamma})$ for all $\gamma$ in $\widehat{G}$. It follows that $\widetilde{\gamma} \in \mathcal{M}_{\Lambda}^{1}(G, X)$. Now since $\Lambda$ is a Riesz set, the measure $\widetilde{\wp}$ is $\lambda$ -continuous. Therefore the representing measure $\mathfrak{\mho}$ of the operator $T$ has relatively compact range since $X$ has type II- $\Lambda$-CCP. This shows that the operator $T$ is compact (see [2, p. 161]). Thus $X$ has type III- $\Lambda$-CCP. The proof is complete.

On the other hand, for a non Riesz subset of $\widehat{G}$, we shall proceed as in [4] to show that the situation is completely different.

Proposition 3.9. Let $\Lambda$ be a non Riesz subset of the dual group $\widehat{G}$ of a compact metrizable abelian group $G$. Then a Banach space $X$ has type III- $\Lambda$-CCP if and only if it has the complete continuity property.

Proof. It is clear that a Banach space with CCP has type III- $\Lambda$-CCP. For the converse, suppose the Banach space $X$ has III- $\Lambda$-CCP, where $\Lambda$ is a non Riesz subset of $\widehat{G}$. Let $S: \mathcal{C}(G) \longrightarrow X$ be an absolutely summing operator. We want to show that $S$ is compact. Let $q: \mathcal{C}(G) \longrightarrow \mathcal{C}(G) / \mathcal{C}_{A^{\prime}}(G)$ be the natural quotient map. Since $\Lambda$ is not a Riesz set, the dual space $\left(\mathcal{C}(G) / \mathcal{C}_{\Lambda^{\prime}}(G)\right)^{*}=\mathcal{M}_{\Lambda}^{1}(G)$ is not separable, and hence $q^{*}\left(\left(\mathcal{C}(G) / \mathcal{C}_{\Lambda^{\prime}}(G)\right)^{*}\right)$ is not separable. Exactly as in the proof of $[\mathbf{4}$, Theorem 11], by a result of H. P. Rosenthal [12], there exists a subspace $Z$ of $\mathcal{C}(G)$ isometric to $\mathcal{C}(G)$ such that the restriction map $\left.q\right|_{Z}: Z \longrightarrow q(Z)$ is an isomorphism. Thus we have the following diagram

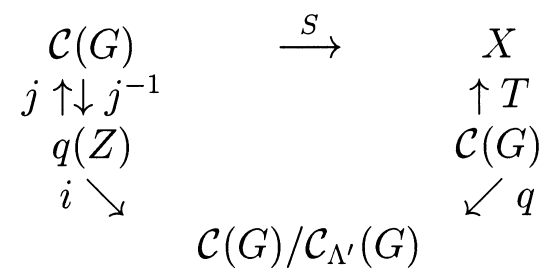

where $j$ is an isomorphism, $i$ is the inclusion map.

Let $\tilde{S}=S j$. Then since $S$ is absolutely summing, $\tilde{S}$ is Pietsch integral (see for example [2, p. 165]). Let $\tilde{T}$ be the Pietsch integral extension of $\tilde{S}$ to $\mathcal{C}(G) / \mathcal{C}_{A^{\prime}}(G)$, and 
define $T=\tilde{T} q$. Then the operator $T$ is Pietsch integral and thus it is absolutely summing. Also $T(f)=\tilde{T}(q(f))=0$ for every function $f \in \mathcal{C}_{\Lambda^{\prime}}(G)$. Since the Banach space $X$ has type III- $\Lambda$-CCP $T$ is compact and so is $T_{\mid Z}=\tilde{T} q_{\mid Z}: Z \longrightarrow X$.

Now $\tilde{S}=\tilde{T}_{\mid q(Z)}=\left(\tilde{T}_{q \mid Z}\right) \circ\left(q_{\mid Z}\right)^{-1}: q(Z) \longrightarrow X$. Thus the operator $\tilde{S}$, and consequently $S=\widetilde{S j}^{-1}$, is compact. The proof is complete.

Let us finish this section with the following interesting result.

THEOREM 3.10. Let $\Lambda$ be a subset of $\hat{G}$. The following properties are equivalent:

(i) $\mathcal{M}_{\Lambda}^{1}(G)$ has $\mathrm{CCP}$;

(ii) $\mathcal{M}_{\Lambda}^{1}(G)$ has RNP.

Proof. We need only show that (i) $\Rightarrow$ (ii). Assume $\mathcal{M}_{\Lambda}^{1}(G)$ has CCP. We claim that this implies $L^{1}(G)$ has III- $\Lambda$-CCP. To see this, let $T: C(G) \rightarrow L^{1}(G)$ be a 1 summing operator such that $T_{\mid C_{\Lambda^{\prime}}(G)}=0$. Let $\tilde{T}: C(G) / C_{\Lambda^{\prime}}(G) \rightarrow L^{1}(G)$ be such that the following diagram commutes.

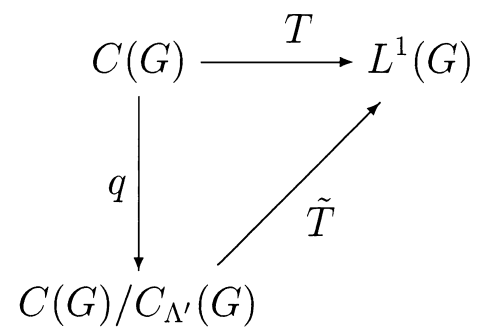

It was pointed out in [4] that since $T$ is Pietsch integral, then it follows from a result of Grothendick [2] that $\tilde{T}$ is also Pietsch integral. Hence $\tilde{T}^{*}: L^{1}(G)^{*} \rightarrow$ $\left(C(G) / C_{\Lambda^{\prime}}(G)\right)^{*}$ is Pietsch integral. Since $\left(C(G) / C_{\Lambda^{\prime}}(G)^{*}\right.$ is isometric to $\mathcal{M}_{\Lambda}^{1}(G)$ and $\mathcal{M}_{\Lambda}^{1}(G)$ is assumed to have CCP, and since Pietsch integral operators factor through $L^{1}$ spaces, it follows that $\tilde{T}$ is compact, hence $T$ is compact. This proves the claim. Moreover, if $L^{1}(G)$ has III- $\Lambda$-CCP, then it follows from Proposition 3.9 that $\Lambda$ should be a Riesz set. This of course implies that $\mathcal{M}_{\Lambda}^{1}(G)=L_{\Lambda}^{1}(G)$ and thus $\mathcal{M}_{\Lambda}^{1}(G)$ has RNP since it is a separable dual Banach space [2].

4. $G_{\delta}$-embedding and concluding remarks. In [7], N. Ghoussoub and H. P. Rosenthal proved the following:

Proposition 4.1. Let $T$ be a bounded linear operator from $L^{1}$ to a Banach space $Y$ and let $S$ be a $G_{\delta}$-embedding of $Y$ into a Banach space $X$. Then the operator $T$ is completely continuous if and only if so is the operator $S T$.

Recall that given two Banach spaces $X$ and $Y$, an element $T \in \mathcal{L}(X, Y)$ is a $G_{\delta^{-}}$ embedding if for any closed subset $F$ of $Y, T(F)$ is a $G_{\delta}$-subset of $Y$.

Proposition 4.1 establishes in particular that the CCP is stable under $G_{\delta^{-}}$ embedding. In this section, we shall see that this result can also be used to prove the stability property of the types I-, II- and III- $\Lambda$-CCP under $G_{\delta}$-embedding, where $\Lambda$ is a subset of the dual group of a compact metrizable abelian group $G$.

The proof of the stability of type I- $\Lambda$-CCP under $G_{\delta}$-embedding is immediate by Proposition 4.1 . 
THEOREM 4.2. Let $\Lambda$ be a subset of the dual group of a compact metrizable abelian group $G$. Let $X$ be a Banach space with type I- $\Lambda$-CCP. Then every Banach space that $G_{\delta}$-embeds in $X$ has type I- $\Lambda$-CCP.

The fact that the II- $\Lambda$-CCP is also stable by $G_{\delta}$-embedding is straight forward as shown in the following theorem.

THEOREM 4.3 Let $\Lambda$ be a subset of the dual group of a compact metrizable abelian group $G$. Let $X$ be a Banach space with type II- $\Lambda$-CCP. Then every Banach space that $G_{\delta}$-embeds in $X$ has type II- $\Lambda$-CCP.

Proof. Suppose that the Banach space $Y G_{\delta}$-embeds in $X$. Let $S: Y \longrightarrow X$ denote the $G_{\delta}$-embedding. Let $\mu \in M_{A a c}^{1}(G, Y)$. Define $v: \mathcal{B}(G) \longrightarrow X$ by $v(A)=S(\mu(A))$, for $A \in \mathcal{B}(G)$. It is easy to see that $v$ is a is $\lambda$-continuous $\Lambda$-measure of bounded variation. Therefore by our hypothesis, the measure $v$ has relatively compact range. On the other hand, by the Hahn decomposition theorem, there exists a sequence $\left(E_{n}\right)$ of disjoint members of $\mathcal{B}(G)$ such that $G=\bigcup_{n=1}^{\infty} E_{n}$ and with the property that for each Borel subset $A$ of $G$

$$
(n-1) \lambda\left(A \cap E_{n}\right) \leq|\mu|\left(A \cap E_{n}\right) \leq n \lambda\left(A \cap E_{n}\right) .
$$

For each positive integer $n$, consider the increasing sequence of measurable subsets of $G$ defined by $\widetilde{E}_{n}=\bigcup_{\nu=1}^{\nu=n} E_{\nu}$. It is clear that $G=\bigcup_{n=1}^{\infty} \widetilde{E}_{n}$, and thus

$$
\lim _{n} \lambda\left(G \backslash \widetilde{E}_{n}\right)=0 .
$$

For each $n \in \mathbb{N}$, let $\mu_{n}$ be the measure defined by $\mu_{n}(A)=\mu\left(A \cap \widetilde{E}_{n}\right)$, for every $A \in \mathcal{B}(G)$. Then by construction the measures $\mu_{n}$ are of bounded average range and as such define bounded linear operators $T_{n}: L^{1}(G) \longrightarrow Y$ by $T_{n}(f)=\int_{G} f d \mu_{n}$, for $f \in L^{1}(G)$. It is clear that for each $n \in \mathbb{N}$, and for every $A \in \mathcal{B}(G)$,

$$
\nu\left(A \cap E_{n}\right)=S T_{n}(A) .
$$

Since the measure $v$ has relatively compact range, we see that the operator $S T_{n}$ is completely continuous. Proposition 4.1 ensures that, for each $n \in \mathbb{N}$, the operator $T_{n}$ is also completely continuous and therefore the measure $\mu_{n}$ has relatively compact range, for each $n \in \mathbb{N}$.

Now for each $n \in \mathbb{N}$, and for every $A \in \mathcal{B}(G)$, we have

$$
\begin{aligned}
\left\|\mu(A)-\mu_{n}(A)\right\| & =\left\|\mu(A)-\mu\left(A \cap \widetilde{E}_{n}\right)\right\| \\
& =\left\|\mu\left(A \cap\left(G \backslash \widetilde{E}_{n}\right)\right)\right\| \\
& \leq\left\|\mu\left(G \backslash \widetilde{E}_{n}\right)\right\| .
\end{aligned}
$$

It follows from (4.1) and (4.2) that $\lim _{n} \mu_{n}=\mu$ uniformly on $\mathcal{B}(G)$. Hence for every $\epsilon>0$, there exists $n_{\epsilon}$ large enough so that

$$
\{\mu(A): A \in \mathcal{B}(G)\} \subset\left\{\mu_{n_{\epsilon}}(A): A \in \mathcal{B}(G)\right\}+\epsilon B(Y) .
$$

Since $\left\{\mu_{n_{\epsilon}}(A): A \in \mathcal{B}(G)\right\}$ is relatively compact for any arbitrary $\epsilon>0$, a standard argument shows that $\{\mu(A): A \in \mathcal{B}(G)\}$ is also relatively compact. This finishes the proof. 
Finally for the case of type III- $\Lambda$-CCP, we saw that this property is equivalent to either: type II- $\Lambda$-CCP, for $\Lambda$ Riesz (see Proposition 3.8), or CCP, for $\Lambda$ non Riesz (see Proposition 3.9). Therefore, we immediately have the following.

THEOREM 4.4. Let $\Lambda$ be a subset of the dual group of a compact metrizable abelian group $G$. Let $X$ be a Banach space with type III- $\Lambda$-CCP. Then every Banach space that $G_{\delta}$-embeds in $X$ has type III- $\Lambda$-CCP.

The next theorem is a known result of J. Bourgain and H. P. Rosenthal [1].

THeOREM 4.5. The sequence space $c_{0} G_{\delta}$-embeds in a Banach space $X$ if and only if it embeds in $X$.

Proof. One implication is obvious. For the other implication suppose $c_{0}$ fails to embed in $X$. Then $X$ has type I- $\Lambda$-CCP for any Sidon set $\Lambda$ by Theorem 2.6, hence $c_{0}$ cannot $G_{\delta}$-embed in $X$.

Finally, we can show the following result.

Proposition 4.6. Let $\Lambda$ be an infinite subset of the dual group $\widehat{G}$ of a compact metrizable abelian group $G$. Then $L^{1}(G) / L_{\Lambda^{\prime}}^{1}(G)$ fails I- $\Lambda$-CCP.

Proof. Let $q: L^{1}(G) \rightarrow L^{1}(G) / L_{\Lambda^{\prime}}^{1}(G)$ be the natural quotient mapping. It is clear that $q(\bar{\gamma})=0$ for any $\gamma \notin \Lambda$, thus $q$ is a $\Lambda$-operator but $q$ is not completely continuous for the sequence $\left(\bar{\gamma}_{n}\right)$ where $\gamma_{n} \in \Lambda$ is a weakly null sequence, yet the sequence $\left\|q\left(\bar{\gamma}_{n}\right)\right\| \geq 1$ for all $n \geq 1$.

In [10], A. Pełczyński showed that if $L^{1}(\mathbb{T}) / H^{1}(\mathbb{T})$ embeds in a Banach lattice $X$, then $X$ must contain an isomorphic copy of $c_{0}$. The following result reveals that in fact the conclusion of the statement of the above proposition remains true for the Banach lattice $X$ if we replace "embeds" by " $G_{\delta}$-embeds" in the statement.

Proposition 4.7. Let $\Lambda$ be a Riesz subset of the dual group $\widehat{G}$ of a compact metrizable abelian group $G$. Then if $L^{1}(G) / L_{\Lambda^{\prime}}^{1}(G) G_{\delta^{-}}$embeds in a Banach lattice $X$, then $X$ must contain an isomorphic copy of $c_{0}$.

Proof. If the Banach lattice $X$ contains no copy of $c_{0}$, then $X$ has type I- $\Lambda$-CCP by Theorem 2.5. If we combine the result of Proposition 4.6 with that of Theorem 4.2, we see that $L^{1}(G) / L_{\Lambda^{\prime}}^{1}(G)$ cannot $G_{\delta^{-}}$-embed in $X$.

\section{REFERENCES}

1. J. Bourgain and H. P. Rosenthal, Applications of the theory of semi-embeddings to Banach Space Theory, J. Funct. Anal. 52 (1983), 149-188.

2. J. Diestel and J. J. Uhl, Jr., Vector measures, Math. Surveys, 15 (AMS, Providence, R.I., 1977).

3. J. Diestel, H. Jarchow and A. Tonge, Absolutely summing operators (Cambridge studies in advanced mathematics 43, 1995).

4. P. Dowling, Radon-Nikodým properties associated with subsets of countable discrete abelian groups, Trans. Amer. Math. Soc. 327 (1991), 879-890. 
5. P. Dowling, Duality in some vector valued function spaces, Rocky Mountain J. Math. 22 (1992), 511-518.

6. G. A. Edgar, Banach spaces with the analytic Radon-Nikodým property and compact abelian groups, Proc. International Conf. on Almost Everywhere Convergence in Probability and Ergodic Theory (Columbus, Ohio), 195-213, Academic Press (1989).

7. N. Ghoussoub and H. P. Rosenthal, Martingales, $G_{\delta}$-embeddings and quotients of $L^{1}$, Math. Ann. 264 (1983) 321-332.

8. E. Hewitt and K. A. Ross, Abstract in harmonic analysis I-II (Springer-Verlag, New York, 1970).

9. F. Lust-Piquard, Proprietes geometriques des sous-espaces invariants par translation de $L^{1}(G)$ et $C(G)$, Seminaire sur la geometrie des espaces de Banach (Ecole Polytechnique, exposé 26, 1977/78).

10. A. Pełczyński, Banach spaces of analytic functions (C.B.M.S. Regional Conference, Kent, Ohio 30, 1977).

11. N. Randrianatoanina and E. Saab, Stability of some types of Radon-Nikodým properties, Illinois J. Math. 39 (1995), 416-430.

12. H. P. Rosenthal, On factors of $\mathcal{C}[0,1]$ with nonseparable dual, Israel J. Math. 13 (1976), 833-835.

13. W. Rudin, Fourier analysis on groups (Interscience Tracts in Math., 12, Interscience Publishers Inc., New York, 1962). 